\title{
Transverse Single Spin and Azimuthal Asymmetries in Hadronic Collisions at STAR
}

\author{
Anselm Vossen, a \\ ${ }^{1}$ Indiana University
}

\begin{abstract}
Hadronic collisions with transversely polarized protons are an important part of the quest to understand the transverse spin structure of the proton. Experiments at RHIC collected large datasets at center of mass energies of $200 \mathrm{GeV}$ and $500 \mathrm{GeV}$, accessing a kinematic regime where factorization is expected to hold. The STAR detector at RHIC, due to its azimuthal symmetry, particle identification capabilities and large acceptance compared with other experiments with polarized protons, is in a unique position to study transverse spin phenomena in $p+p^{\uparrow}$. This contribution will highlight measurements of transversity using di-hadron correlations, transverse single spin asymmetries in jets, measurements sensitive to the origins of the large single spin asymmetries measured in the forward direction, transverse spin asymmetries in $\mathrm{W}$ production, which are sensitive to modified universality effects of Sivers function as well as short and long-term upgrades at STAR.
\end{abstract}

\section{Introduction}

The study of transverse single spin asymmetries (TSSAs) in $p+p^{\uparrow}$ collisions has a long history (see [1] and references therein). Perhaps the most well known result is that large TSSAs are observed for pions and charged kaons in the forward region. Here the forward direction is loosely defined as events with an $x_{F}>0.2$, where $x_{F}=\frac{p_{L}}{2 \sqrt{s}}$. At leading order, for 2-2 scattering, $x_{F}=x_{1}-x_{2}$, where $x_{i}$ are the $\mathrm{x}$ values of the partons participating in the scattering. Large $x_{F}$ therefore corresponds to the valence quark region, where experiments have shown that transverse spin effects are large. The quest to understand the origin of the large transverse spin asymmetries has motivated substantial progress in the theory community. Notably is the progress in leading twist models that take intrinsic transverse momentum in the parton distribution (PDF) or fragmentation function (FF) into account [2][3] and calculations that go beyond leading twist in the nucleon structure or the fragmentation process [4][5]. In the following I will call the former the TMD picture and the latter the twist3 picture. The TMD picture is valid in processes with two scales, one hard scale that allows to factorize long range from short range physics and a soft scale for the intrinsic $k_{T}$. In $p+p$ hadroproduction, the $p_{T}$ is usually the hard scale, whereas for electroweak production (W production and Drell-Yan) the mass of the produced $\mathrm{W}$ or $\gamma^{*}$ indicates the momentum transfer in the hard scattering. The lower scale $\left(k_{T}\right)$ for hadroproduction is given by the transverse momentum of detected hadrons with respect to the jet direction if one is looking at final state effects (i.e. coming from fragmentation) or by the di-jet imbalance if one is

\footnotetext{
a e-mail: avossen@indiana.edu
}

looking at initial state effects. For electroweak production it is the transverse momentum of the produced vector boson. In the twist 3 picture, there is only one hard scale, which is usually given by the $p_{T}$ of the detected jet or hadron. It should be noted, that it was recently shown, that for processes with more than two color-charged objects involved, factorization is broken for the TMD picture [6]. For these proceedings, the most relevant example of this, are processes which lead to azimuthal modulations of hadrons in jets. The advantage of the TMD picture is, that it allows for an easier separation of the underlying physics effects, since it usually involves the correlation between the large and small scale momenta and the nucleon spin and momentum. In contrast, the twist 3 framework only uses one scale, so the observables have to be constructed with only one final state momentum vector in hand.

These developments show that $p+p$ collisions provide access to QCD dynamics that are very difficult to access in other experiments. Furthermore, the many measurements are complementary to physics programs using Semi Inclusive Deep Inelastic Scattering (SIDIS). Collisions can be much harder at a $p+p$ collider operating at the center of mass energies that RHIC does. In $p+p$ collisions, gluonic degrees of freedom can be accessed directly and there is no u-quark dominance as for measurements using electromagnetic probes. Furthermore, experiments at a $p+p$ collider are sensitive to a wide range of scattering angles, therefore they are able to access valence quarks at relatively high values of $x$ as well as sea-quarks. These proceedings will describe some of the unique measurements done by the STAR collaboration at RHIC to explore the transverse spin structure. Section 2 will give an 
overview of the STAR experiment with an emphasis on the capabilities that make it unique for spin measurements in $p+p$. The following four sections focus on the measurement of the collinear transverse spin structure (section 3), TMD observables(section 4 ), very forward single scale measurements (section 5) and TMD observables in electroweak-production (section 6).

\section{The STAR Experiment at RHIC}

The Solenoidal Tracker at RHIC (STAR) [7] detector at the Relativistic Heavy Ion Collider (RHIC) is unique since its comparatively large acceptance allows the reconstruction of jets in polarized proton-proton collisions. It also covers a kinematic range, where transverse spin effects generate significant signals. Since 2002, RHIC delivered polarized proton beams to the STAR interaction region. Significant samples were collected at $200 \mathrm{GeV}$ and $500 \mathrm{GeV}$ center of mass energies $\sqrt{s}$. At these energies, momentum transfers can be selected at which the leading twist structure of the proton dominates the measurement. The solid angle instrumented can be roughly segmented in three parts: The so-called mid-rapidity region covers pseudorapidity, $\eta$ values from about -1 to 1 . Here using the STAR Time Projection Chamber (TPC) and Barrel Electromagnetic Calorimeter (BEMC) jets can be reconstructed as well as charged particles. PID capabilities are provided by $\mathrm{dE} / \mathrm{dx}$ and time-of-flight measurements. The endcap region which covers roughly $1.1<\eta<2.0$ is instrumented by the STAR endcap electromagnetic calorimeter [8] and partially by the TPC. However, as the angle of tracks becomes steeper at higher values of $\eta$, the number of points on the tracks decreases, which leads to a worse momentum resolution and worse $\mathrm{dE} / \mathrm{dx}$ measurements. Realistically, tracks up to $\eta=1.3$ can be reconstructed. With the planned inner sector TPC upgrade, addressed in section 7 , the readout density of the TPC will be increased significantly, so that the whole endcap region will be covered by tracking. Current analyses at STAR use $\pi^{0}$ and $\eta$ mesons reconstructed in the endcap, as well as jets from electromagnetic clusters (EM-jets). Finally, the forward region at STAR with $2.7<\eta<4.0$ is instrumented by the Forward Meson Spectrometer (FMS) where again neutral mesons and EM-jets can be reconstructed. It should be noted, that the physical $\eta$ can be signficantly different from the socalled detector $\eta$ due to the longitudinal spread of the vertex distribution.

\section{Measuring the Collinear Transverse spin Structure of the Proton with Di-hadron Correlations}

Of the three Parton Distribution Functions, the so-called transversity $h(x)$ describes the transverse spin structure of the nucleon [9]. Due to its chiral-odd nature, it is the least well known PDF and can only be measured in conjunction with another chiral-odd function. The interference fragmentation function (IFF) is a chiral-odd fragmentation function, describing the spin dependent production of unpolarized hadrons from transversely polarized quarks. In $p+p$ an observable can be formulated in a colinear, leading twist framework that is directly sensitive to the product of $h(x)$ with the IFF $H^{\varangle}(z, M)$. Here $z$ is the fractional energy of the parent quark the produced hadron pair is carrying and $M$ the invariant mass of the hadron pair. Following [10], the polarized cross-section for charged pion pair production in a $p+p^{\uparrow}$ collision can be written in a simplified form as

$$
\begin{aligned}
\sigma_{U T} \propto & \sum_{a, b, c, d}|\vec{S}| \sin \left(\Phi_{S}-\Phi_{R}\right) . \\
& \int \frac{d x_{a} d x_{b}}{z_{c}} f\left(x_{b}\right) h\left(x_{a}\right) \frac{d \hat{\sigma}_{a^{\uparrow} b \rightarrow c^{\uparrow} d}}{d \hat{t}} H^{\varangle}\left(z, M^{2}\right) .
\end{aligned}
$$

Here $\vec{S}$ is the spin vector of the polarized proton, $f(x)$ is the unpolarized PDF, $a, b, c, d$ the partons participating in the 2-2 scattering, $\Phi_{S}$ the azimuthal angle of the spin vector with respect to the scattering plane, $\Phi_{R}$ the azimuthal angle of the vector $\vec{R}=\vec{P}_{h 1}-\vec{P}_{h 2}$ with respect to the scattering plane around $\vec{P}_{h}=\vec{P}_{h 1}+\vec{P}_{h 2}, \vec{P}_{h 1,2}$ are the momenta of the two hadrons, $\hat{t}$ the partonic Mandelstam variable and $M$ the invariant mass of the hadron pair. To extract $h(x) H^{\varangle}(z, M)$ and cancel the detector acceptance effects, we form the spin asymmetry

$$
A_{U T}^{\sin \Phi} \sin \left(\Phi_{R S}\right)=\frac{1}{P} \frac{N^{\uparrow}\left(\Phi_{R S}\right)-R N^{\downarrow}\left(\Phi_{R S}\right)}{N^{\uparrow}\left(\Phi_{R S}\right)-R N^{\downarrow}\left(\Phi_{R S}\right)}
$$

Here $\Phi_{R S}=\Phi_{S}-\Phi_{R}, P$ the polarization of the beam, $R$ the relative luminosity between the up and down spin states and $N^{\uparrow, \downarrow}$ the number of di-hadron pairs in a specific $\Phi_{R S}$ bin where the polarized beam in up ( $\left.\uparrow\right)$ or down $(\downarrow)$. The amplitude of the $\sin \left(\Phi_{R S}\right)$ modulation $A_{U T}^{\sin \Phi}$ is then directly proportional to transversity times the IFF normalized by the unpolarized cross-section. At STAR $A_{U T}^{\sin \Phi}$ has been measured using a dataset with an integrated luminosity of $1.8 \mathrm{pb}^{-1}$ taken in 2006 using charged identified pion pairs at mid-rapditity. Figure 1 shows the measured values for $A_{U T}^{\sin \Phi}$ vs $\eta$. From simulations using GEANT and Pythia, we estimate that mean $x$ for the datapoints reach from 0.15 to 0.25 . The mean $z$ is about 0.4 . The rise in the mean $x$ indicates that at higher $\eta$ valence quarks participate in the scattering, which carry significant transversity. These results are the first direct probe of transversity in $p+p$. Datasets taken at $\sqrt{s}=200 \mathrm{GeV}$ in 2012 and at $\sqrt{s}=500 \mathrm{GeV}$ in 2011 that are currently being analyzed have about an order of magnitude more statistics and will therefore increase the precision of this measurement significantly.

\section{Physics with Jets}

Jets in $p+p$ collisions can be used to access the TMD structure of the proton [11]. Here, the jet axis serves as a proxy of the outgoing parton. Therefore, we can measure the dependence of the intrinsic transverse momentum generated in the fragmentation process on the parent proton spin. This can be used to access transversity via the 


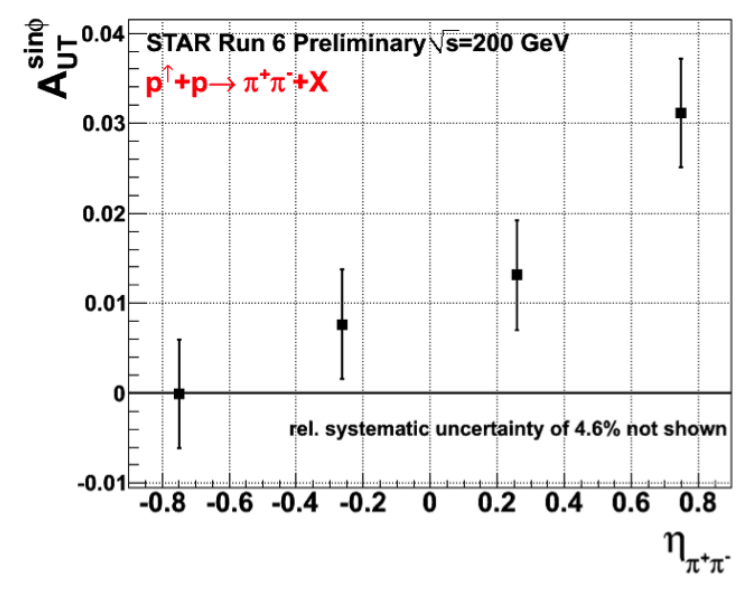

Figure 1. Di-hadron Transverse Spin Asymmetries vs $\eta$ measured at mid-rapdity. The lowest $\eta$ corresponds to an $x$ of 0.15 , whereas the highest corresponds to $x=0.25$. The rise of the measured asymmetries with $\eta$ is consistent with the rising magntitude of transversity over this $x$-range.

TMD Collins fragmentation function, or to access the linear polarization of gluons in the protons. Furthermore, jet kinematics provide access to the underlying partonic kinematics assuming 2-2 scattering due to the relation $x_{1,2}=\frac{1}{\sqrt{s}}\left(p_{T 1} e^{ \pm \eta_{1}}+p_{T 2} e^{ \pm \eta_{2}}\right)$, where the $p_{T i}, \eta_{i}$ are the transverse momenta and rapidities of the detected jets and $x_{1,2}$ the lightcone fractions of the partons. Even if only one jet is detected, this means that high $p_{T}$ and large $\eta$ are more sensitive to the valence region. The transverse spin asymmetries measured in jet production is similar to eq. 2 , only that the angle $\Phi_{R}$ is replaced by the azimuthal angle $\Phi_{h}$, which is the azimuthal angle of the identified pion in the jet measured around the jet axis with respect to the scattering plane. The scattering plane is now spanned by the beam and the jet axis. The Collins effect, where transversity couples to the Collins Fragmentation function $H\left(z, \vec{k}_{T}\right)$ similarly to the IFF measurement, can then be measured as a $\sin \left(\Phi_{S}-\Phi_{h}\right)$ modulation, whereas the linear gluon polarization leads to a $\sin \left(\Phi_{S}-2 \Phi_{h}\right)$ modulation. These are so called Collins-like Asymmetries. Both of these observables are interpreted in the TMD picture, which means that the interpretation will suffer from the problems described in the introduction. A measurement with jets, that has only one hard scale, is the azimuthal modulation of the jet itself. That means one extracts the dependence of the jet crosssection on $\sin \left(\Phi_{S}\right)$, the azimuthal angle between the proton spin and the produced jet. Clearly, this measurement is insensitive to final state effect. In the twist 3 framework, which is appropriate due to the single hard scale, this observable is sensitive to $T_{F}$, a twist3 quark-gluon correlator. In turn, $T_{F}$ is related to the Sivers function through an integral relationship [12][13]. At STAR we measured Collins-like asymmetries at $500 \mathrm{GeV}$. Here glue-glue scattering dominates at mid-rapidity, in particular at low jet $p_{T}$. Figure 2 shows the STAR results. We measured very small asymmetries. Since this is the first time that this

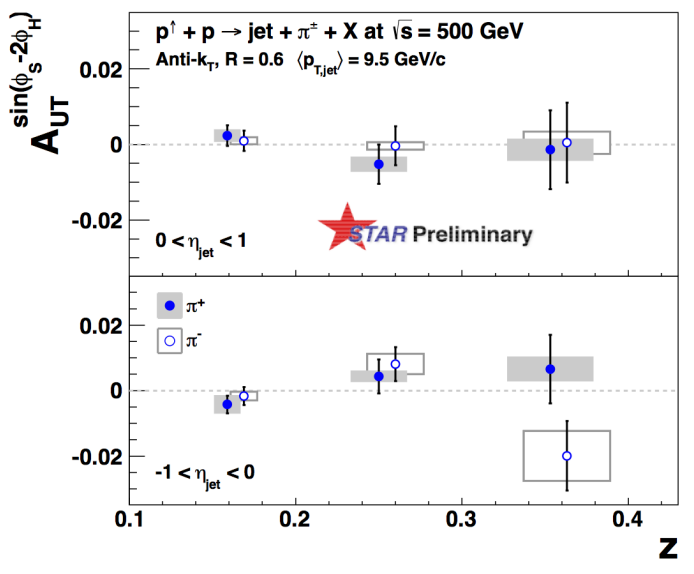

Figure 2. Collins-like asymmetries at $500 \mathrm{GeV}$ for low $p_{T}$ vs $z$. Shown are the data identified charged pions for low $p_{T}$, where the gluon contributions are enhanced. For both, forward and backward asymmetries, the asymmetries are small, which will provide bounds for model calculations.

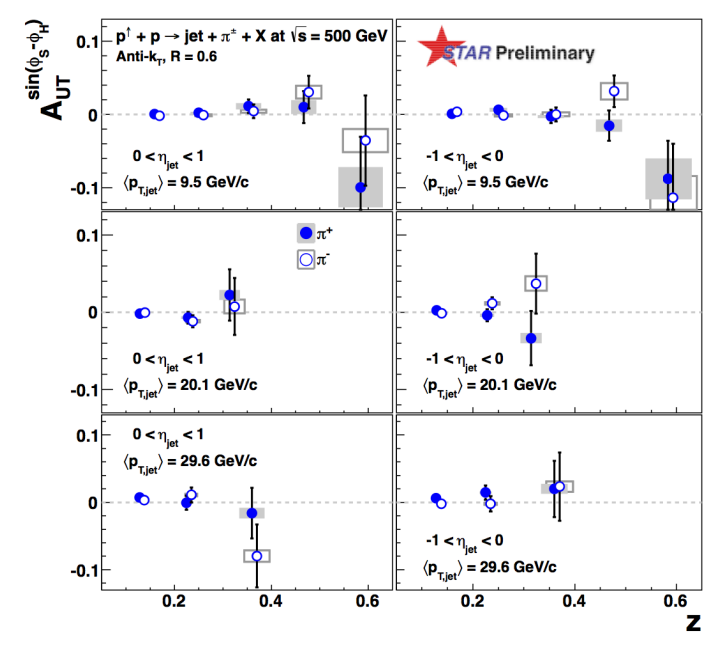

Figure 3. Collins asymmetries at $500 \mathrm{GeV}$. Shown are forward $\eta>0$ and backward $\eta<0$ asymmetries for different ranges in jet $p_{T}$. Throughout the kinematic region accessed, the asymmetries are small for $\pi^{+}$and $\pi^{-}$.

quantity has been meaured, the STAR results will provide important limits for model calculations.

Collins asymmetries have been extracted from the same dataset at $\sqrt{s}=200 \mathrm{GeV}$ used for the di-hadron asymmetry measurement. The results have been presented before [14]. The results are consistent with zero due to the large error bars, however, from calculations [15] which assume that factorization breaking effects are negligible it is expected that the larger dataset already taken at $200 \mathrm{GeV}$ will reduce the statistical errors enough to result in a significant result.

Collins asymmetries have also been measured at $500 \mathrm{GeV}$. Results are shown in fig. 3. However, since transversity for gluons vanishes, it is expected that Collins asymmetries are small for the region covered by STAR. 


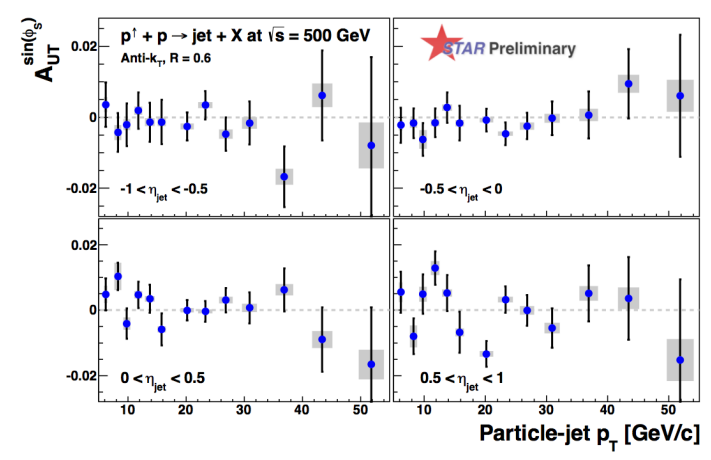

Figure 4. Jet $A_{N}$ at $500 \mathrm{GeV}$. Small asymmetries are observed over the full range of $p_{T}$ at all rapidities accessed in the STAR barrel. This bounds the magnitude of the twist 3 correlator $T_{F}$ and the related Sivers function in this kinematic region, which is dominated by gluon scattering.

Consistent with this, the STAR results do not show significant asymmetries. The $\sqrt{s}=500 \mathrm{GeV}$ dataset has also been used to extract transverse spin asymmetries of jets, which are sensitive to the gluon Sivers function. Results are shown in fig. 4. Asymmetries are very small, consistent with a small gluon Sivers function. (Full results presented at [16])

\section{Forward Single Spin Asymmetries}

As described in section 1, the study of forward transverse spin asymmetries $A_{N}$ for neutral pions have a long history in $p+p$ scattering. Similar to the observable for jets described in section $4, A_{N}$ is a very simple observable [17]. It is proportional to the excess of particles that go to left vs. the right if the spin of the polarized proton points up. Due to its simplicity it is relatively hard to pin down the separate contributions to the asymmetry, since unlike the jet $A_{N}$, both initial and final state effects can play a role. Most measurements have been done with reconstructed $\pi^{0}$ mesons, but also $\eta$ mesons [1] as well as charged pions and kaons have been measured [18]. STAR observed large asymmetries for $\pi^{0}$ at large transverse momenta in $\sqrt{s}=500 \mathrm{GeV}$ collisions. To investigate the mechanisms behind these asymmetries, STAR measured asymmetries for EM-jets for different photon multiplicities. In particular, transverse single spin asymmetries $A_{N}$ for jets from photons reconstructed in the FMS in $2.7<\eta<4.0$ with an anti- $k_{T}$-algorithm with radius of $R=0.7$ are measured. Figure 5 shows the result for $A_{N}$ vs. jet energy for jets consisting of two photons in the invariant mass region of the $\pi^{0}$. This jet sample consists therefore mainly of isolated $\pi^{0}$. The magnitude of these asymmetries rises with the energy and is consistent with the forward $A_{N}$ measured for inclusive $\pi^{0} \mathrm{~s}$.

In contrast, asymmetries for jets with more than 2 photons are significantly smaller and show a flat dependence on the jet energy. A possible conclusion from these results is that the contribution of initial state effects is negligible for the large forward $A_{N}$. That the asymmetry is large

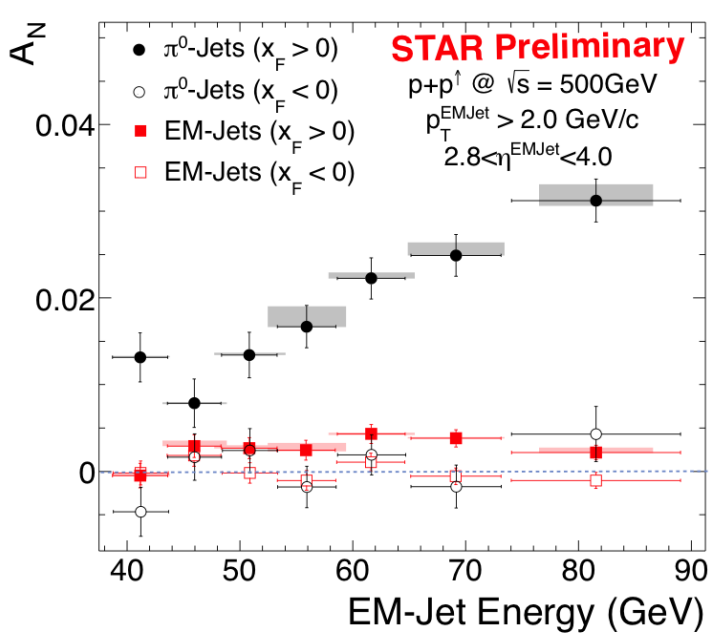

Figure 5. Forward EM-jets vs. jet-energy. Jets in the backward direction $\left(x_{F}<0\right)$ are consistent with zero. Asymmetries for jets consisting of two photons that are in the $\pi^{0}$ mass region are consistent with the forward $A_{N}$ measured for $\pi^{0} \mathrm{~s}$ at other experiments. In contrast, jets with a higher photon count, have significantly smaller asymmetries.

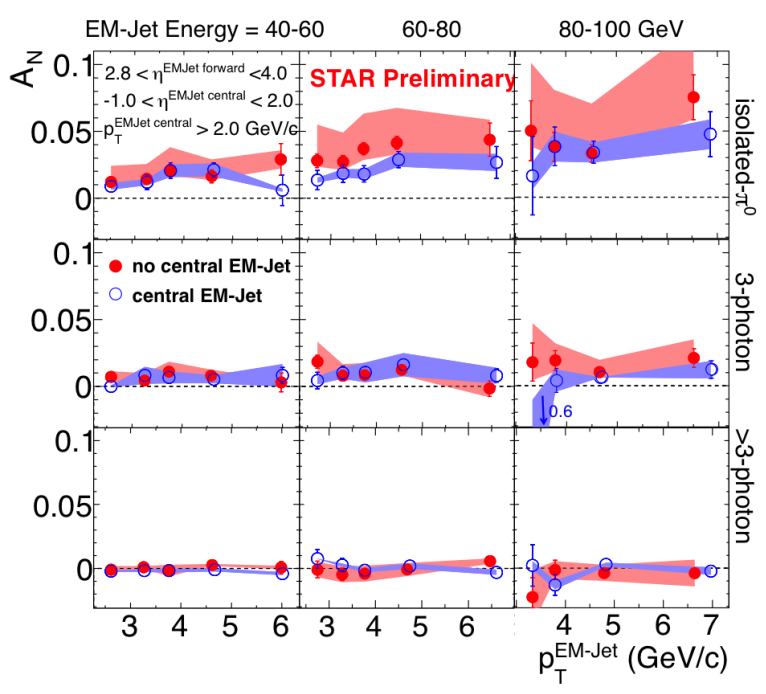

Figure 6. Forward EM-jets with and without central jet requirement vs. EM-jet $p_{T}$ and multiplicity. The requirement of jet at midrapidity or the endcap region leads to significantly lower asymmetries for isolated $\pi^{0}$.

for isolated $\pi^{0}$ could mean that exclusive processes contribute to $A_{N}$. To test this hypothesis, we repeated the same measurement for events where in addition to the forward EM-jet, a EM-jet in the mid-rapidity or endcap region of STAR was detected and for events, where no EM-jet was found at mid-rapidity or in the endcap. Figure 6 shows that for the 2-photon case (isolated $\pi^{0} \mathrm{~s}$ ) the requirement of a jet at mid-rapidity leads to a significant decrease of the asymmetries. For jets with larger multiplicities, the difference is within the statistical and systematic errors, since the signal is very small. Even though this result seems 
to indicate, that exclusive processes might play a significant role for single transverse spin asymmetries at high $\eta$ in $p+p$, it has to be noted, that the requirement of the second jet at mid-rapidity restricts the kinematic region of the measurement compared to the case where none is detected. In the latter case the second jet might have been in a region that is not instrumented. However as noted above, in 2-2 scattering the larger $x$ accessed can be computed as $x=\frac{1}{\sqrt{s}}\left(p_{T 1} e^{\eta_{1}}+p_{T 2} e^{\eta_{2}}\right)$. This means, that for events, where jets are missed because they fall into a region which is not instrumented at negative $\eta, x$ remains virtually unchanged. The case where the undetected jet falls into the forward region (i.e. $\eta>4.0$ ) has a negligible cross-section. This suggests, that hard 2-2 scattering where both outgoing partons fragment into jets are not the leading contributor to the large $A_{N}$.

\section{Transverse Single Spin Asymmetries of fully reconstructed $W$ Bosons}

One of the milestones in the nuclear physics community is to observe the sign change of the Sivers function between processes with final state interactions (FSI), like SIDS, vs processes with initial state interaction (ISI). ISI processes can be probed in hadronic collisions, like polarized $p+p$. Two quarks annihilate and form a $\gamma^{*}$ or a $\mathrm{W}$ or $\mathrm{Z}$ boson. One then has to pick out the process where the electroweak gauge boson decays leptonically, to isolate the ISI. The leptonic decay of the $\mathrm{W}$ boson at STAR has a very unique signature. Since the $\mathrm{W}$ has high mass, W produced even at mid-rapidity probe the Sivers function in the valence region as well as the sea-region. For this analysis, fully reconstructed Ws are used. The momentum of the undetected neutrino is reconstructed from the recoil which is corrected for particles escaping through holes in the detector using MC simulations. Figure 7 shows the results from the first exploratory measurement in 2011. The statistical error bars are still too large to test the predicted sign change of the Sivers function. However, this measurement used only an integrated luminosity of $25 \mathrm{pb}^{-1}$, which has to be compared with the luminosity collected during a potential long run at $\sqrt{s}=500$ at RHIC that could collect as much as $900 \mathrm{pb}^{-1}$. With this amount of statistics, this channel will be very valuable in testing the sign change predictions for the Sivers function even the evolution from SIDIS to the $\mathrm{W}$ scale introduces some uncertainty. In addition to the sign change, this measurement will be very important to determine the Sivers function for sea-quarks, which are virtually unconstrained at the moment as well as the QCD evolution of the Sivers function [19].

\section{Short and longterm Upgrades}

STAR plans a number of short and long term upgrades that will extend the reach of its spin program [20]. For the next RHIC run, Run15, starting at the end of this year, STAR will have a preshower detector for the FMS in place. The main physics goal of this detector is the measurement of transverse spin asymmetries of direct photons which are
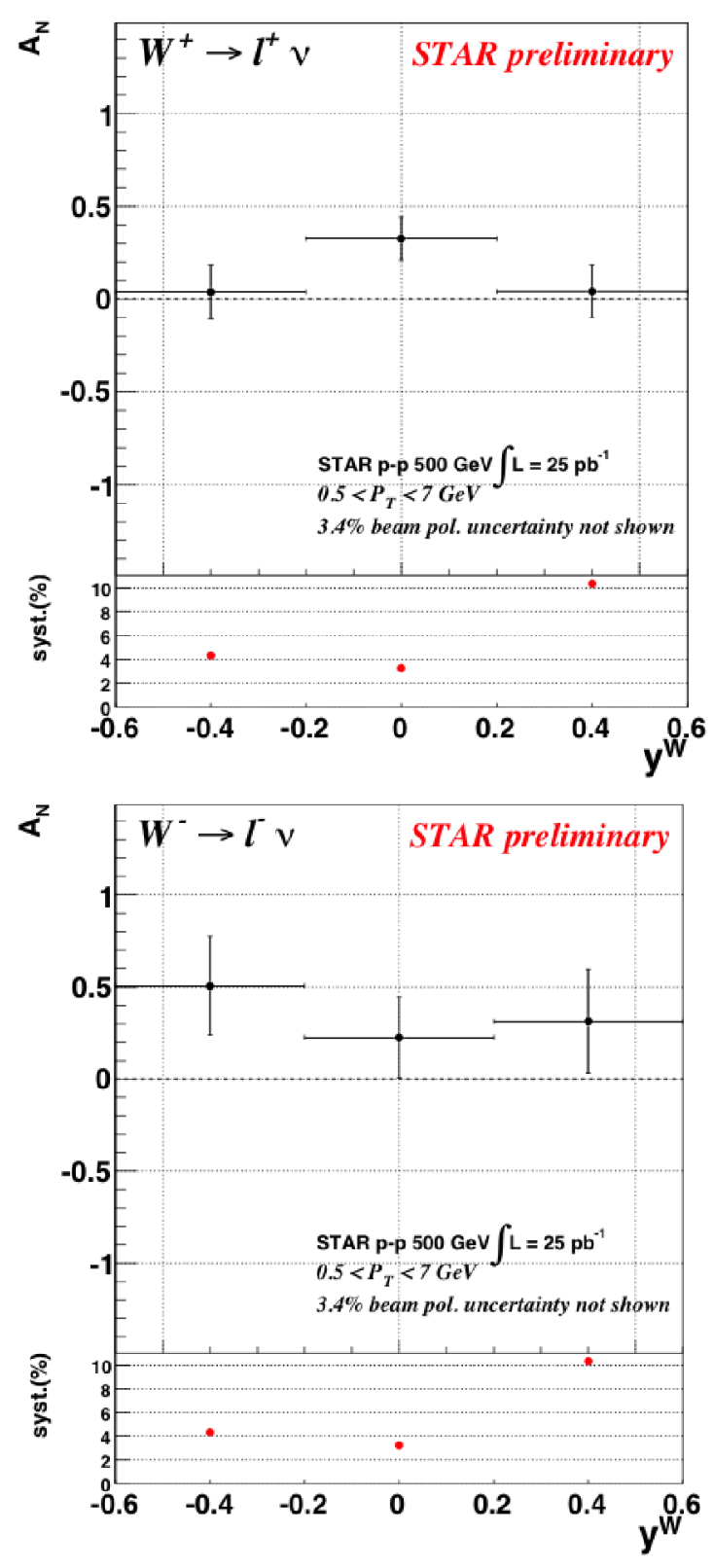

Figure 7. Transverse single spin asymmetry $A_{N}$ for fully reconstructed $\mathrm{W}$ bosons at midrapidity vs. W pseudorapidity. The statistical error bars of this exploratory measurements are still too large, to give an answer to the Sivers sign-change question. However, they will provide limits on the sea-quark Sivers function.

again related to the Sivers function. Since photons do not carry color charge, the relevant calculations are well understood. In addition, RHIC will for the first time have polarized $p+A$ collisions in Run15. Probing high gluon densities at low $x$ in Au with spin can provide additional insight into the physics of the saturation regime. In the long term, possibly starting around 2020, STAR is planning for a more extensive forward upgrade. The centerpiece of which is a new combined ECAL/HCAL system covering $2.5<\eta<4.5$ that will allow jet reconstruction in the forward region as well as hadron reconstruction. A tracking system is also planned to allow charge reconstruc- 
tion which is needed e.g. for the di-hadron correlation measurements. Possible physics topics with transverse spin include precision measurements of Collins and Sivers asymmetries as well as IFF asymmetries. This would allow the measurement of transversity up to values of $x$ of 0.4 and greater. It could also address question about the TMD evolution of the Collins function and possible factorization breaking effect. Another upgrade, which is currently underway, is the instrumentation of inner sectors of the TPC. Currently only about a third of the inner readout pads are instrumented, which means that for tracks at steeper angles, not enough points can be reconstructed for tracking or PID. As mentioned above, currently the tracking capability of STAR ends at about $\eta=1.3$. With the inner sector upgrade, tracking will be available up to $\eta=2.0$, therefore covering the STAR endcap. This would enable a similar phyisic program than in the STAR barrel, but at higher $x$. It is expected that this upgrade will be finished by the end of 2017 .

\section{References}

[1] A. Adare et al. (PHENIX Collaboration) (2013), 1312.1995

[2] M. Anselmino, Transverse spin physics: overview (2014), in these proceedings

[3] S. Melis, Phenomenology of TMDs (2014), in these proceedings

[4] D. Pitonyak, Twist-three in fragmentation and its impact on $A_{N}$ (2014), in these proceedings

[5] Y. Koike, The role of three-gluon correlation functions in the single spin asymmetry (2014), in these proceedings

[6] T.C. Rogers, P.J. Mulders, Phys.Rev. D81, 094006 (2010), 1001.2977
[7] K. Ackermann et al. (STAR Collaboration), Nucl.Instrum.Meth. A499, 624 (2003)

[8] C. Allgower et al. (STAR Collaboration), Nucl.Instrum.Meth. A499, 740 (2003)

[9] V. Barone, A. Rago, P.G. Ratcliffe, Phys.Rept. 359, 1 (2002), hep-ph/0104283

[10] A. Bacchetta, M. Radici, Phys.Rev. D70, 094032 (2004), hep-ph/0409174

[11] U. D’Alesio, F. Murgia, C. Pisano, Phys.Rev. D83, 034021 (2011), 1011.2692

[12] D. Boer, P. Mulders, F. Pijlman, Nucl.Phys. B667, 201 (2003), hep-ph/0303034

[13] X. Ji, J.W. Qiu, W. Vogelsang, F. Yuan, Phys.Rev.Lett. 97, $082002 \quad$ (2006), hep-ph/0602239

[14] R. Fatemi (STAR Collaboration), AIP Conf.Proc. 1441, 233 (2012), 1206. 3861

[15] C. Pisano, Azimuthal Asymmetries in $p^{\uparrow} p \rightarrow$ jet $\pi X$ (2014), in these proceedings

[16] J. Drachenberg, Constraining Transversity and $\mathrm{Nu}$ cleon Transverse-polarization Structure Through Polarized Proton Collisions at STAR (2014), presented at PANIC 2014 - Particles and Nuclei International Conference 2014

[17] G.G. Ohlsen, P.W. Keaton, Nucl. Instrum. Meth. 109 (1973)

[18] J. Lee, F. Videbaek (BRAHMS Collaboration) (2009), 0908.4551

[19] M.G. Echevarria, A. Idilbi, Z.B. Kang, I. Vitev, Phys.Rev. D89, 074013 (2014), 1401.5078

[20] The STAR Collaboration, STAR Note SN0605, https://drupal.star.bnl.gov/STAR/starnotes/public/sn0605 (2014) 